\title{
Crosshole seismic tomography with cross-firing geometry
}

\author{
Ying Rao', Yanghua Wang ${ }^{2}$, Shumin $\mathrm{Chen}^{3}$, and Jianmin Wang ${ }^{3}$
}

\begin{abstract}
We have developed a case study of crosshole seismic tomography with a cross-firing geometry in which seismic sources were placed in two vertical boreholes alternatingly and receiver arrays were placed in another vertical borehole. There are two crosshole seismic data sets in a conventional sense. These two data sets are used jointly in seismic tomography. Because the local sediment is dominated by periodic, flat, thin layers, there is seismic anisotropy with different velocities in the vertical and horizontal directions. The vertical transverse isotropy anisotropic effect is taken into account in inversion processing, which consists of three stages in sequence. First, isotropic traveltime tomography is used for estimating the maximum horizontal velocity. Then, anisotropic traveltime tomography is used to invert for the anisotropic parameter, which is the normalized difference between the maximum horizontal velocity and the maximum vertical velocity. Finally, anisotropic waveform tomography is implemented to refine the maximum horizontal velocity. The cross-firing acquisition geometry significantly improves the ray coverage and results in a relatively even distribution of the ray density in the study area between two boreholes. Consequently, joint inversion of two crosshole seismic data sets improves the resolution and increases the reliability of the velocity model reconstructed by tomography.
\end{abstract}

\section{INTRODUCTION}

We present a case study of crosshole seismic tomography. An interesting feature of this crosshole seismic acquisition is its cross-firing fashion between two vertical boreholes. Basically, there are two sets of crosshole seismic data in a conventional sense. The first data set places sources in one borehole and the receiver arrays in another. The second data set swaps sources and receiver arrays with the opposite boreholes. These two data sets form a cross-firing geometry and are used jointly in seismic inversion.

The local sediment is dominated by periodic flat thin layers (Figure 1). This type of geologic structure may cause vertical transverse isotropy (VTI) anisotropy, in which the horizontal velocity component is faster than the vertical velocity component (Thomsen, 1986). The geophysical objectives of this crosshole seismic study are (1) to generate a high-resolution image to facilitate identification of the thin layers and (2) to extract an anisotropic parameter that is suitable for surface seismic processing. The anisotropy parameter is defined by the normalized difference between the maximum horizontal and vertical velocities. Seismic tomography attempts to present an image for the following two model elements: the maximum horizontal velocity and the anisotropic parameter. Because these two model parameters have different physical units, different magnitudes, and different sensitivities, they can be inverted sequentially in traveltime tomography.

Crosshole traveltime tomography uses the first-arrival times to reconstruct the velocity model. However, because two boreholes are so close together, picking errors in traveltime data may not be treated as random variables with a Gaussian distribution and the least-squares solution may often be biased. In crosshole seismic data, the signal-to-noise ratio likely depends on the vertical offset between a source and a receiver. The smaller the vertical offset is, the more certain the observation should be, because the overall attenuation will typically be smaller for a shorter raypath and hence the distinctness and strength of the arrival increase. It follows logically that a taper function can be set inversely based on the vertical offset, and be applied to data fitting in the isotropic traveltime tomography (Berryman, 1989; Rao and Wang, 2005). Such a

\footnotetext{
Manuscript received by the Editor 4 December 2015; revised manuscript received 16 February 2016; published online 13 May 2016.

${ }^{1}$ China University of Petroleum (Beijing), State Key Laboratory of Petroleum Resource \& Prospecting, Beijing, China, and Centre for Reservoir Geophysics, Department of Earth Science and Engineering, Imperial College London, London, UK. E-mail: raoying@cup.edu.cn.

${ }^{2}$ Centre for Reservoir Geophysics, Department of Earth Science and Engineering, Imperial College London, London, UK. E-mail: yanghua.wang @imperial .ac.uk.

${ }^{3}$ Daqing Oilfield Company Ltd., Research Institute of Exploration and Development, Daqing, China. E-mail: chenshumin@petrochina.com.cn; jianminwang@petrochina.com.cn.

(C) 2016 Society of Exploration Geophysicists. All rights reserved.
} 
weighting scheme is a direct reflection of confidence in the accuracy of the picked traveltime data. Another argument given for such weights is that the raypaths with small vertical offset are more likely to correspond to real raypaths that are controlled predominantly by the maximum horizontal velocity. Consequently, the isotropic traveltime tomography with this weighting scheme produces the maximum horizontal velocity model.

Once this horizontal velocity model is obtained, anisotropic traveltime tomography is used to estimate the anisotropy parameter, by gradually relaxing the weighting enforced in large source-receiver (vertical) offsets. For crosshole seismic, Chapman and Pratt (1992) and Pratt and Chapman (1992) develop linear systems for 2D traveltime tomography in anisotropic media, and Pratt et al. (1993) and Pratt and Sams (1996) show that the anisotropic velocity tomography is a valuable tool to detect the discontinuities in the investigation region. Zhou et al. (2008) use a nonlinear inversion method for Thomsen's anisotropic parameters from the traveltime inversion. Rao and Wang (2011) demonstrate that the anisotropic traveltime tomography results in a much better match in the first-arrival times between the synthetic and observed data, particularly at far offsets.

If ignoring the existence of anisotropy, crosshole seismic tomography would commonly have X-type artifacts in the velocity image, produced by either in the traveltime tomography (Rao and Wang, 2005) or waveform tomography (Wang and Rao, 2006). The weighted taper function, with respect to source-receiver (vertical) offsets, applied to crosshole seismic data can also suppress the common X-type artifacts in these isotropic inversions. The X-type artifacts link the top and the bottom corners of the study area between two boreholes. They exist in these largest offsets because there are less data to average the local solution. This is an evidence of velocity anisotropy, because the directional velocity along raypaths of the largest offsets is far different from an averaged velocity along raypaths of modest offsets. These observations just indicate

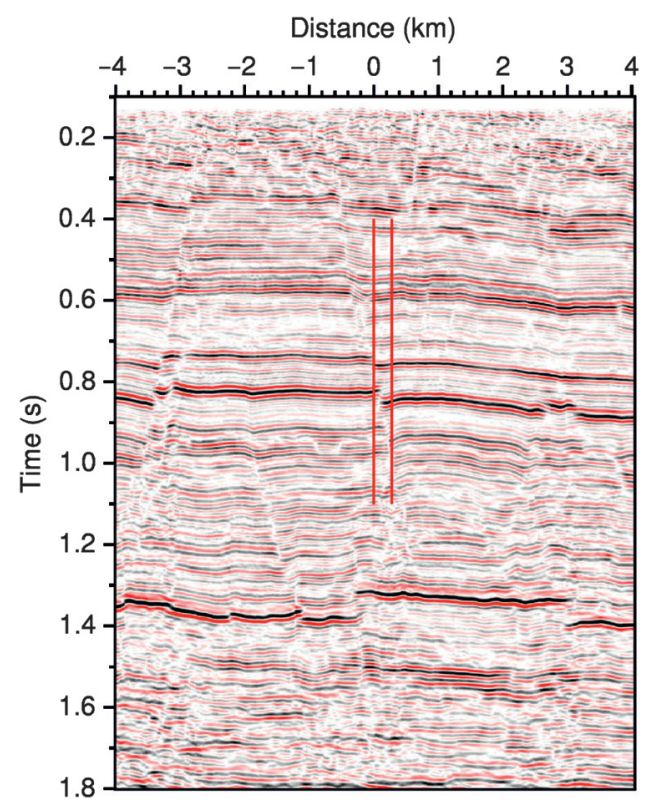

Figure 1. A seismic line crossing two boreholes, showing that the local geologic structure is dominated by periodic, flat, and thin-layered sediments. Two vertical boreholes (red lines) have a separation of $230 \mathrm{~m}$. that the anisotropic effect should be considered in reconstructing a velocity model through tomographic inversion.

In anisotropic waveform tomography, if the anisotropy parameter can be assumed to be a constant only in the background, it may be preset as a constant shrink factor in the whole investigation area during the numerical calculation (Pratt and Shipp, 1999). In this paper, however, a 2D anisotropic model is explicitly defined in seismic simulation with an anisotropic wave equation, instead of a simple shrinking factor working on finite-differencing grids. In summary, we present a three-stage inversion procedure: (1) isotropic traveltime tomography, inverting for the maximum horizontal (group) velocity model, (2) anisotropic traveltime tomography, inverting for the anisotropic parameter, and (3) anisotropic waveform tomography, refining the maximum horizontal (group) velocity model, or inverting for the maximum horizontal (phase) velocity model.

Anisotropic waveform tomography is implemented in the frequency domain, taking advantage of its efficiency. Waveform tomography using only distinct frequency components, other than all frequencies of the data, can reconstruct a reliable velocity image. In theory, following the linearity of the Fourier transform, all frequency components of the data set should be used in waveform tomography, for the equivalency of a time-domain full waveform inversion (Warner et al., 2013). However, even if only distinct frequency components are selected for the waveform tomography, the reconstructed velocity model can be comparable with the result of full waveform tomography (Pratt, 1990; Pratt and Worthington, 1990; Zhou and Greenhalgh, 2003; Ravaut et al., 2004; Sirgue and Pratt, 2004; Wang and Rao, 2009). In the frequency domain, the wave equation discretized with a finite-differencing method is formulated as a linear system in a matrix-vector form. The forward modeling operator is decomposed, for solving the linear system, and decomposed matrix factors can be reused to rapidly solve the forward problem for multiple sources. It is especially important in the iterative solution in which many forward solutions for real sources and virtual sources are required (Pratt et al., 1998; Wang, 2011). However, only when the VTI anisotropy is considered in waveform tomography could the first arrivals of the field observation be matched by synthetics, generated with an anisotropic wave equation explicitly constituted by two model elements (the maximum horizontal velocity and the anisotropic parameter) for a weak anisotropic case.

The cross-firing acquisition geometry significantly improves the ray coverage between two boreholes and leads to improvement on the inversion resolution. Consequently, there is a relatively balanced distribution of the ray density in the study area. In traveltime tomography, the slowness (reciprocal velocity) update is linear to the ray density. In waveform tomography, this relationship cannot be presented so straightforwardly, but the model update at any location is also proportional to the ray density (Rao et al., 2006). Therefore, the cross-firing data geometry also increases the reliability of the velocity model reconstructed by tomography.

\section{CROSSHOLE SEISMIC WITH CROSS-FIRING GEOMETRY}

The rationale for designing a cross-firing acquisition geometry is to improve the ray coverage over the study region between two vertical boreholes. Because of the practicality, one often has a very dense receiver array in a borehole but very sparsely positioned sources in another borehole. The ray coverage next to the receiver 
borehole is sufficiently full, but the ray coverage next to the source borehole is terribly poor. Thus, a cross-firing geometry improves ray coverage next to both boreholes.

As shown in Figure 1, the two vertical boreholes are parallel and are $230 \mathrm{~m}$ apart. In the seismic acquisition, source arrays were placed alternatingly in two parallel boreholes (well A on the left and then well $\mathrm{B}$ on the right), and receiver arrays in the opposite borehole ( $\mathrm{B}$ and then $\mathrm{A}$ ). In each borehole, small explosive charges were fired successively at roughly $18 \mathrm{~m}$ intervals in depth. For a single shot gather, a string of six receivers at $6 \mathrm{~m}$ spacing was placed in the other borehole and then repositioned to a different depth to extend the receiver coverage. During repositioning of the receiver string, one receiver point was overlapped for depth calibration and the shot was repeated at the fixed depth.

Figure 2 displays the distribution of the straight-ray density in this physical experiment. This measurement of illumination directly reflects the resolving power in the imaging and the inversion. Figure 2a shows the ray density when sources are placed in well A (on the left side) and receivers are placed in well B (on the right side). In this case, there are 44 receivers and only 14 sources. Because of the limited number of sources with irregular and large depth intervals, many areas close to source well A have a poor ray coverage. Figure $2 b$ is the alternating case in which the sources are placed in well $\mathrm{B}$ and receivers are placed in well $\mathrm{A}$. In this second case, which has 15 sources, the ray density is also unevenly distributed. Figure $2 \mathrm{c}$ suggests that combining these two cases can significantly improve the final ray coverage. This is the motivation for us using both data sets jointly in the following tomographic inversion.

Figure 3a displays two shot gathers at a depth of $1290 \mathrm{~m}$ in well $\mathrm{A}$ and $1300 \mathrm{~m}$ in well B (and the receivers are in the opposite borehole). Both shot gathers clearly show first arrivals with well correlation between traces at different receiver depths. Figure $3 \mathrm{~b}$ displays shot gathers at a depth of $1350 \mathrm{~m}$ in well A and $1340 \mathrm{~m}$ in well B. Because the near-offset traces are missing, we use a smooth curve to fit picked first-arrival times. This curve verifies the shot position in depth, at which the first-arrival time curve has the minimum.

These two groups of shot gathers indicate a strong anisotropic feature. In each group, two shots have $10 \mathrm{~m}$ difference in depth. For the isotropic case, the difference of the first-arrival times would be approximately

$$
\Delta t\left(z-z_{0}\right) \approx \frac{\left(z-z_{0}\right) \Delta z}{v \sqrt{x^{2}+\left(z-z_{0}\right)^{2}}},
$$

where $x=230 \mathrm{~m}$, the distance between two vertical boreholes, $z-$ $z_{0}$ is the vertical offset of a shot-receiver pair, and $\Delta z= \pm 10 \mathrm{~m}$ is the difference between two shot depths. For the group shown in Figure $3 \mathrm{~b}$, for instance, where $z-z_{0}=175 \mathrm{~m}$, if $v=3000 \mathrm{~m} / \mathrm{s}$, we would have $\Delta t \approx \pm 20 \mathrm{~ms}$. However, the difference in two shot records is much smaller than this evaluation because a wave travels with fast directional velocities everywhere along a raypath.

\section{ANISOTROPIC TRAVELTIME TOMOGRAPHY}

Because the local geologic structure is featured with periodic, flat, thin layers, we assume the velocity of the subsurface media to be a simple elliptical anisotropy. The directional velocity $V(\theta)$ is angle dependent, in which the ray angle $\theta$ is measured against the vertical axis. It can be in any form as long as this directional velocity $V(\theta)$ along a raypath can be properly presented (Backus, 1962; Gassmann, 1964; Thomsen, 1986; Alkhalifah, 1998; Fomel, 2004). The directional velocity in a simple elliptical form can be expressed as

$$
V(\theta)=V_{\mathrm{v}} \cos ^{2} \theta+V_{\mathrm{h}} \sin ^{2} \theta,
$$

where $V_{\mathrm{v}}$ is the maximum vertical component of the $\mathrm{P}$-wave velocity when the ray angle $\theta=0$, and $V_{\mathrm{h}}$ is the maximum horizontal velocity when $\theta=\pi / 2$. Defining an anisotropy parameter by
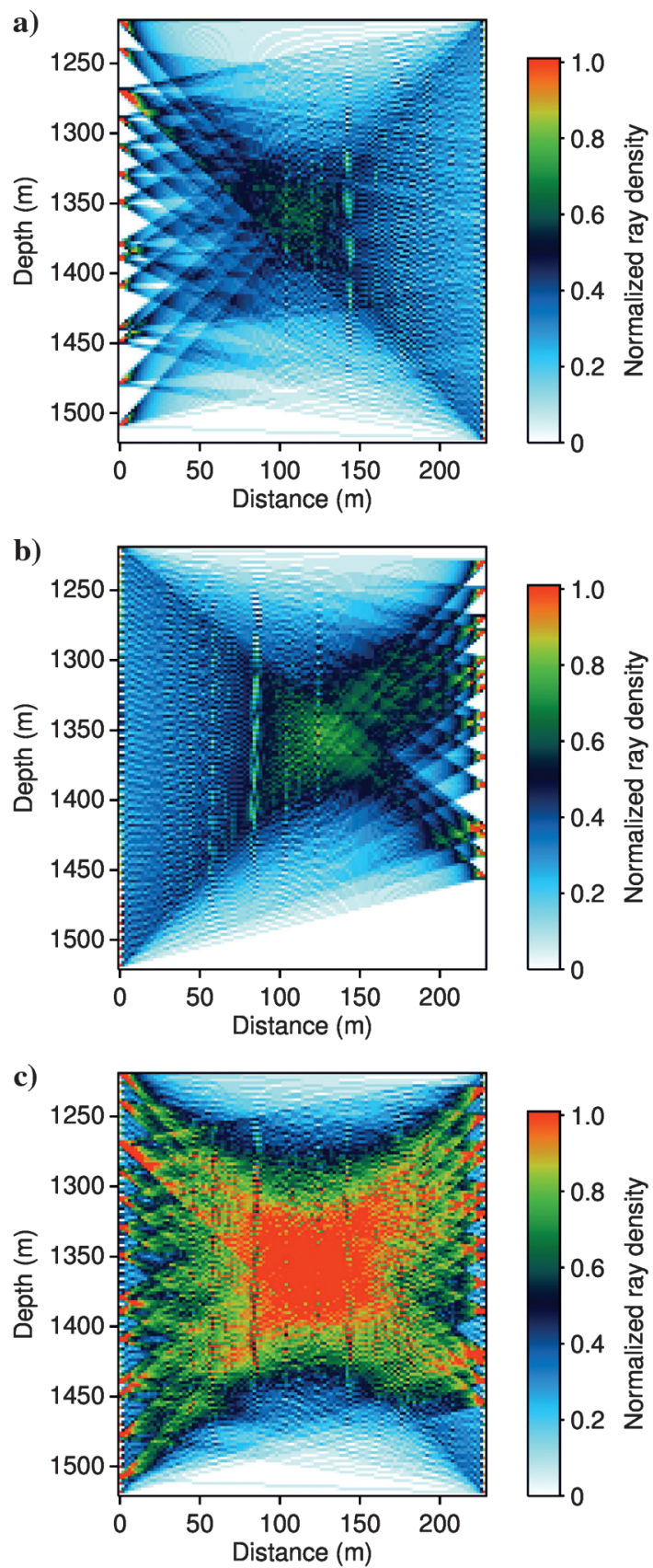

Figure 2. (a) Distribution of ray density, for sources in well A and receivers in well $\mathrm{B}$. (b) Distribution of ray density, for sources in well $\mathrm{B}$ and receivers in well A. (c) Final distribution of ray density, when combining two measurements. 


$$
\varepsilon=\frac{V_{\mathrm{h}}-V_{\mathrm{v}}}{V_{\mathrm{v}}},
$$

equation 2 may also be rewritten as

$$
V(\theta)=V_{\mathrm{v}}\left(1+\varepsilon \sin ^{2} \theta\right)=V_{\mathrm{h}}\left(\frac{1+\varepsilon \sin ^{2} \theta}{1+\varepsilon}\right) .
$$

We use the second expression in which the ray-angle-dependent velocity $V(\theta)$ is determined in terms of the maximum horizontal velocity $V_{\mathrm{h}}$ and the anisotropy parameter $\varepsilon$.

The traveltime between a source-receiver pair is

$$
T=\sum_{i} \Delta t_{i}\left(V_{\mathrm{h}}, \varepsilon\right)
$$

where $\Delta t_{i}$ is the traveltime between two points $\left(x_{i}, z_{i}\right)$ and $\left(x_{i+1}, z_{i+1}\right)$ and $T$ is the traveltime along a raypath. According to Fermat's principle, a raypath has the minimal traveltime; that is,

$$
\nabla T=0
$$

which leads to a system of nonlinear equations. This nonlinear system in anisotropic media has much stronger nonlinearity than the counterpart in isotropic media. In an isotropic case, any perturbation of a raypath causes velocity changes along the perturbed raypath and these velocity changes further affect the raypath. In anisotropic media, any path perturbation causes changes in directional velocities, and these changes depend not only on the spatial position but also on the local propagation direction. Wang (2014) points out that a standard Newton-type iterative algorithm, which relies on the minimization of the errors in the nonlinear system, does not work for anisotropic cases due to the high nonlinearity, and proposes to enforce Fermat's minimum-time principle as a constraint
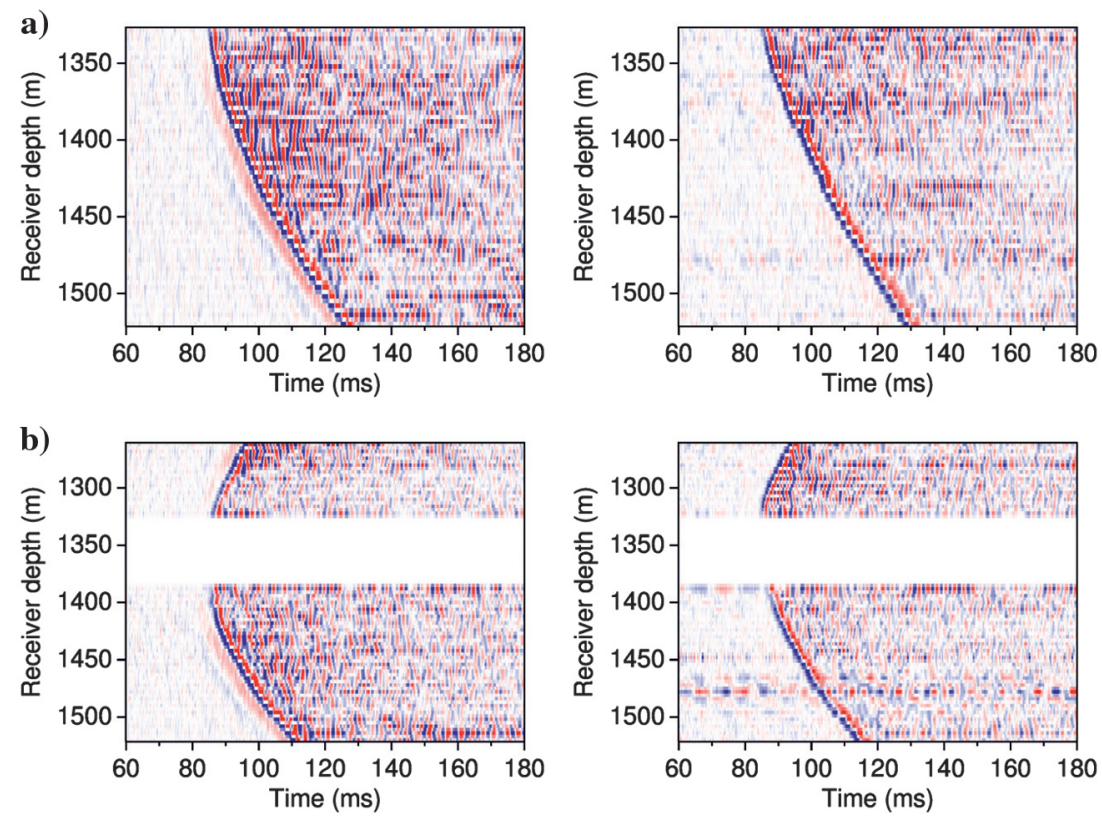

Figure 3. (a) Shot gathers of source number 11 at the depth of $1290 \mathrm{~m}$ in well A and $1300 \mathrm{~m}$ in well B. (b) Shot gathers of source number 8 at the depths of $1350 \mathrm{~m}$ in well A and $1340 \mathrm{~m}$ in well $\mathrm{B}$. for Newton's iterative procedure. Enforcing a physical principle into the solution update of nonlinear algebraic equations significantly stabilizes the iterative procedure, even in complicated anisotropic cases.

In this paper, we do not distinguish between the phase velocity and the group velocity in ray tracing throughout the study area between two vertical boreholes, because these two parallel boreholes are so close to each other. When considering the exact phase velocity, Wang (2013) suggests to replace the raypath concept with the concept of slowness paths, and to solve a system of equations, consisting of slowness equations and an explicit normal constraint that slowness vectors are perpendicular to wavefronts.

In isotropic traveltime tomography for the maximum horizontal velocity model, mainly the small vertical-offset data that contain less of an anisotropic effect are used. Different weights are assigned to traveltimes with different vertical offsets: Weight 1 is in the central range with near offsets and is gradually tapering off with the increase in offsets. That is, near-offset arrival times play a dominant role in the inversion for the maximum horizontal velocity model $V_{\mathrm{h}}$. The initial model is built by assuming raypaths linking sourcereceiver pairs to be straight lines. Back propagation of traveltime residuals generates a rough velocity model (Figure $4 a$ ). This velocity model is refined (Figure $4 \mathrm{~b}$ ) by isotropic traveltime tomography with properly curved raypaths.

To invert for the anisotropy parameter $\varepsilon$, fixing the maximum horizontal velocity $V_{\mathrm{h}}$, all of the available traveltime data with different (vertical) offsets should be used effectively. Figure 5 shows the residuals of the first-arrival times. Figure 5a shows the residuals after traveltime tomography without considering the anisotropic parameter $\varepsilon$, and Figure $5 \mathrm{~b}$ shows the improvement when we take into account the anisotropic effect.

Figure 6 displays two shot gathers (at 1270 and $1380 \mathrm{~m}$ depth in well A) after traveltime tomography (1) without and (2) with the anisotropy parameter, respectively. Once we include the velocity anisotropy effect, the modeled data can better match the field first-arrival times (in the black curve).

Traveltime tomography reveals that an average $\varepsilon$ value between two boreholes is 0.143 ; that is, the average ratio of the maximum horizontal velocity $V_{\mathrm{h}}$ to the maximum vertical velocity $V_{\mathrm{v}}$ is 1.143 . This quantity should be used in the processing and imaging of surface seismic data in this region.

\section{ANISOTROPIC WAVEFORM TOMOGRAPHY}

\section{Anisotropic wave equation}

For anisotropic waveform tomography, we derive an anisotropic wave equation. The wavenumber is defined in terms of velocity and frequency as

$$
k=\frac{\omega}{v},
$$

where $\omega$ is the angular frequency, and $v$ is the phase velocity, and 


$$
v(\phi)=V_{\mathrm{h}}\left(\frac{1+\varepsilon \sin ^{2} \phi}{1+\varepsilon}\right),
$$

expressed in terms of the phase angle $\phi$ and the maximum horizontal velocity $V_{\mathrm{h}}$. Note that equation 8 is an exact phase velocity, and equation 4 is an approximation using the group velocity $V$ and the ray angle $\theta$.

Assuming the following weak anisotropy,

$$
1+\varepsilon \sin ^{2} \phi \approx \sqrt{1+2 \varepsilon \sin ^{2} \phi}
$$

equation 7 can be rewritten as

$$
k=\frac{\omega(1+\varepsilon)}{V_{\mathrm{h}} \sqrt{1+2 \varepsilon \sin ^{2} \phi}} .
$$

Substituting $\sin ^{2} \phi=k_{x}^{2} / k^{2}$ and $k^{2}=k_{x}^{2}+k_{z}^{2}$, we obtain an equation,

$$
\frac{(1+2 \varepsilon) k_{x}^{2}+k_{z}^{2}}{(1+\varepsilon)^{2}}=\frac{\omega^{2}}{V_{\mathrm{h}}^{2}} .
$$
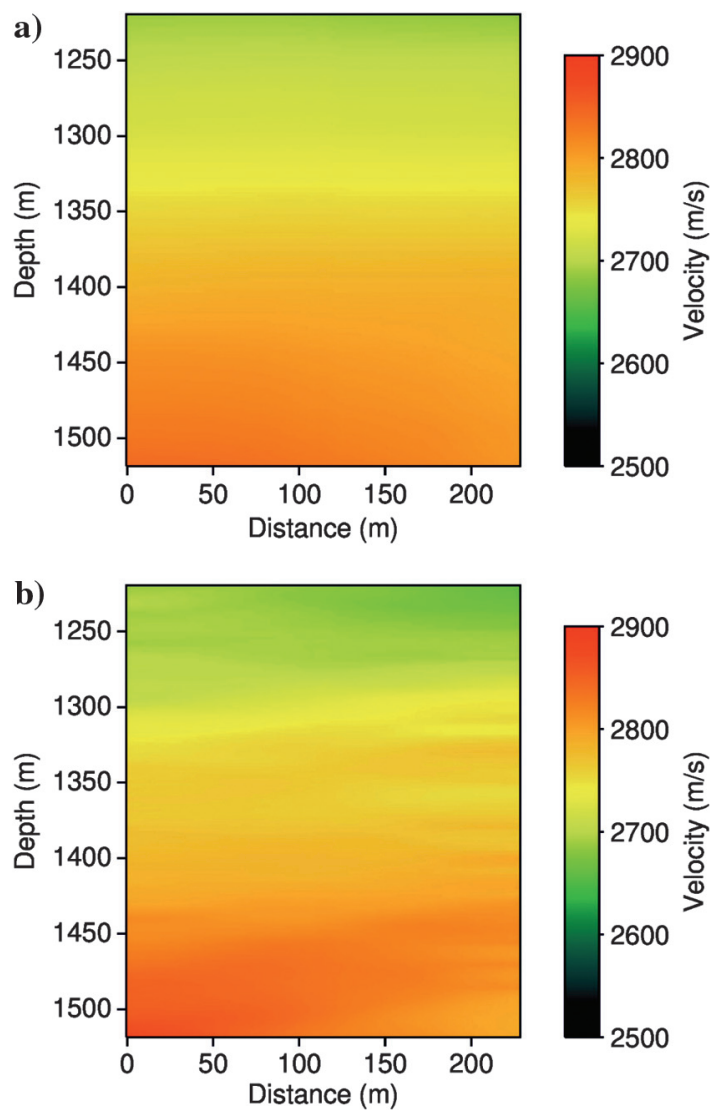

Figure 4. (a) The initial velocity model for traveltime tomography, generated by the error back projection. (b) Velocity model reconstructed by traveltime tomography.
Assuming $(1+2 \varepsilon) /(1+\varepsilon)^{2} \approx 1$ in the case with small $\varepsilon$, we finally obtain

$$
k_{x}^{2}+\frac{1}{(1+\varepsilon)^{2}} k_{z}^{2}-\frac{\omega^{2}}{V_{\mathrm{h}}^{2}}=0 .
$$

A wave equation may be expressed in the wavenumber-frequency domain as

$$
\left(k_{x}^{2}+\frac{1}{(1+\varepsilon)^{2}} k_{z}^{2}-\frac{\omega^{2}}{V_{\mathrm{h}}^{2}}\right) u\left(k_{x}, k_{y}, \omega\right)=0,
$$

a)
Residual time (ms)

b)

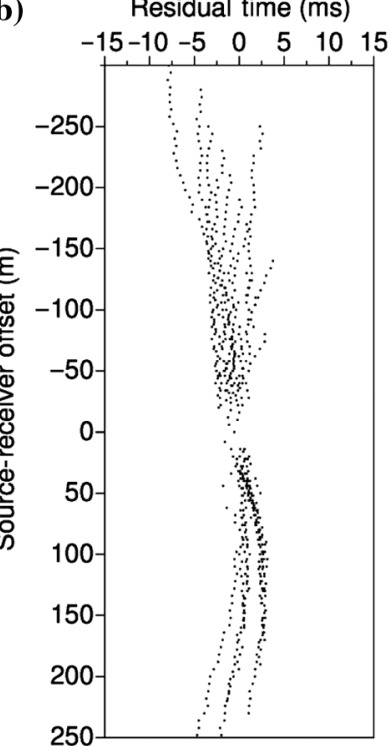

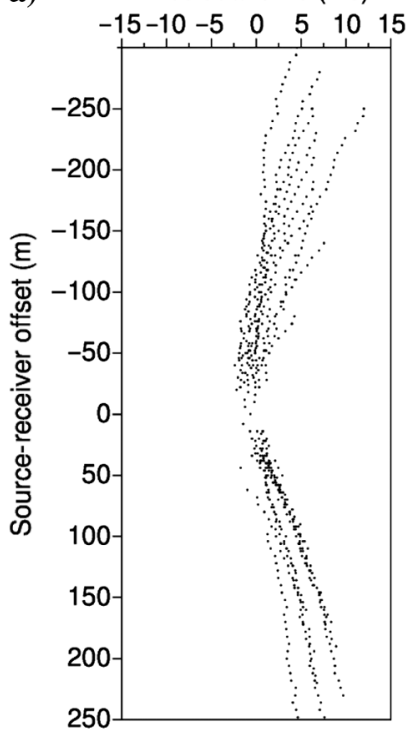

Residual time (ms)

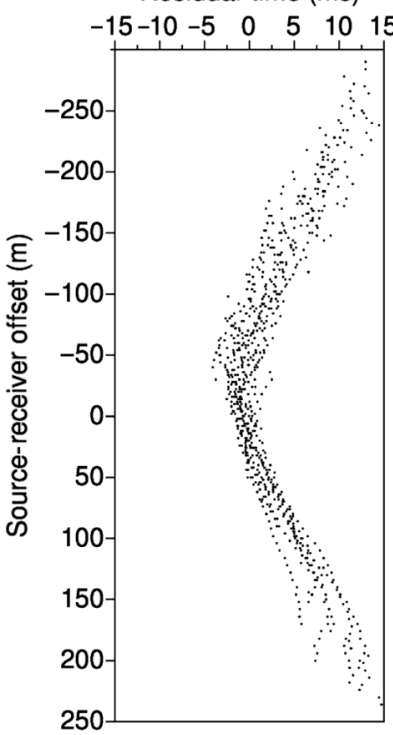

Residual time (ms)

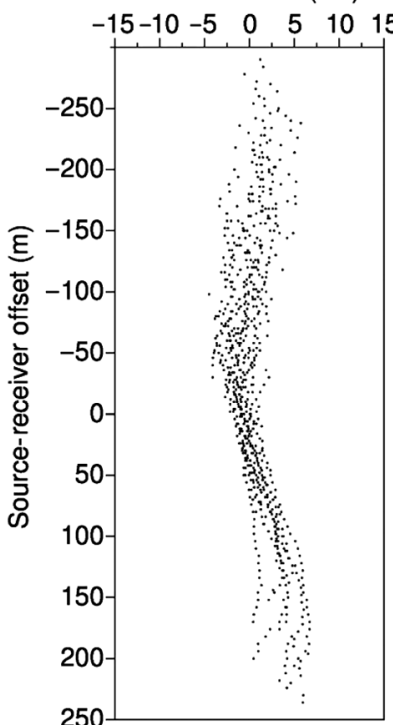

Figure 5. Time residuals after traveltime tomography. (a) The residuals after traveltime tomography without considering the anisotropy parameter. (b) The residuals after traveltime tomography with the anisotropy parameter. 
where $u\left(k_{x}, k_{y}, \omega\right)$ is the wavefield in the wavenumber-frequency domain. The inverse Fourier transformation, with respect to $k_{x}$ and $k_{z}$, respectively, leads to the following space-frequency domain wave equation:

$$
\left(\frac{\partial^{2}}{\partial x^{2}}+\frac{1}{(1+\varepsilon)^{2}} \frac{\partial^{2}}{\partial z^{2}}+\frac{\omega^{2}}{V_{\mathrm{h}}^{2}}\right) u(x, z, \omega)=0 .
$$

After the finite-differencing approximation, we may present this equation in a vector-matrix form, $\mathbf{A x}=\mathbf{b}$. The inversion involves solving the inverse of matrix A. An LU decomposition solver is used in the work reported in this paper. The frequency-domain implementation involves only one of such a matrix decomposition and
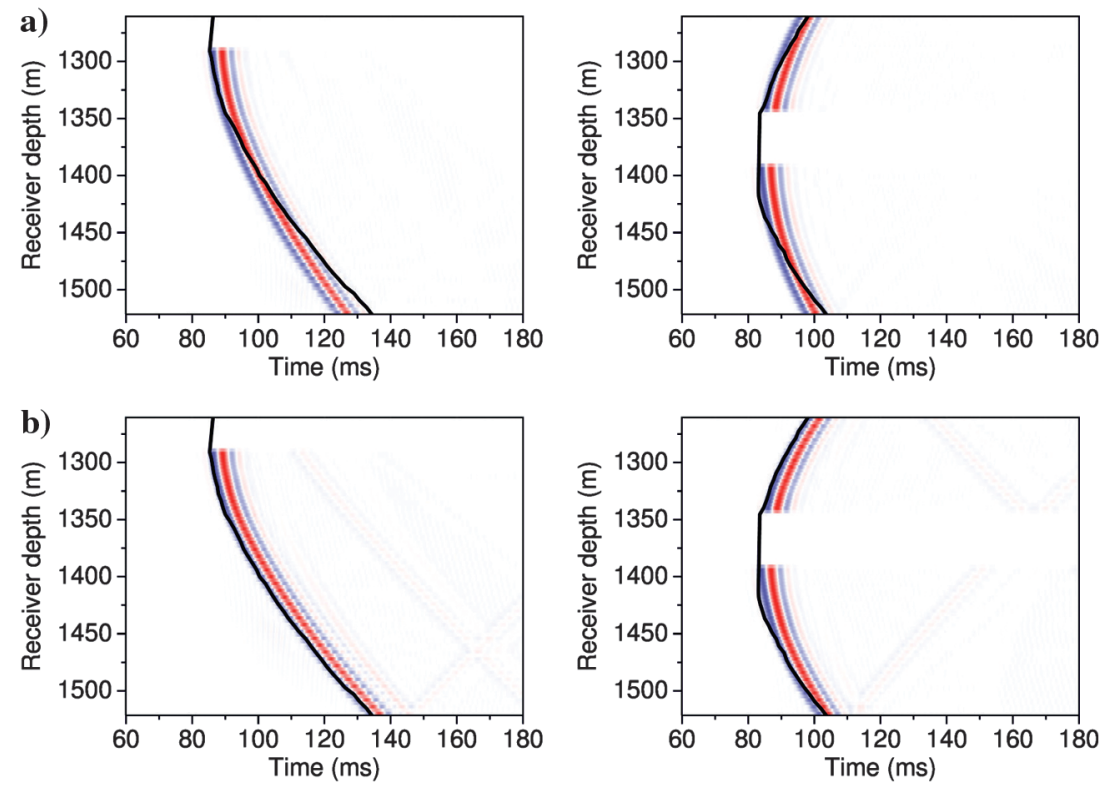

Figure 6. (a) Modeled shot gathers at depths of 1270 and $1380 \mathrm{~m}$ (in well A), based on the traveltime tomography without anisotropy, do not match the field first-arrival times of shot gathers (in the black curve). (b) Modeled shot gathers, based on the traveltime tomography with anisotropy, showing a better match to the field first-arrival times. then it can efficiently calculate a significant number of source locations with negligible cost.

\section{Anisotropic waveform tomography}

In anisotropic waveform tomography, we use the group velocity $V_{\mathrm{h}}(x, z)$ obtained from ray-tracing-based traveltime tomography (Figure $4 \mathrm{~b}$ ) as the initial model $V_{\mathrm{h}}(x, z)$ for the tomography. This is justified between two vertical boreholes that are close to each other. For weak anisotropy, it can be shown (Červený, 2001) that

$$
v(\phi)=V(\theta)\left[1+\frac{1}{2 V^{2}}\left(\frac{\partial V}{\partial \theta}\right)^{2}\right] .
$$

The second term in brackets is rather small and can be neglected, resulting in $v(\phi) \approx V(\theta)$, in contrast to the exact relationship $v(\phi)=$ $V(\theta) \cos (\theta-\phi)$.

Figure 7 displays the amplitude spectrum of a shot gather at a depth of $1290 \mathrm{~m}$ in well A (and receivers in well $\mathrm{B}$ ). It shows that there is no energy for frequencies lower than $100 \mathrm{~Hz}$. Thus, we choose a starting frequency of $100 \mathrm{~Hz}$ for the inversion. For waveform inversion, we discretize the velocity model into cells with cell size of $2 \mathrm{~m}$, to satisfy the criterion of four cells per wavelength for the highest frequency used in the inversion: $v_{\min }=$ $2400 \mathrm{~m} / \mathrm{s}, f_{\max }=300 \mathrm{~Hz}$, and $(1 / 4) v_{\min } / f_{\max }=$ 2 . We choose the depth range to be inverted from 1220 to $1520 \mathrm{~m}$. Therefore, in total there are 115 rows and 150 columns in the grid.

Because the seismic data in the frequency domain have a poor signal-to-noise ratio, a small group of frequency components (usually three to five) simultaneously, instead of using single frequency at a time, is used in waveform tomography. This frequency grouping strategy is for mitigating the effect of data noise, which is not necessarily white in the frequency domain (Pratt and Shipp, 1999; Wang and Rao, 2006). Simultaneously using neighboring frequencies from the same spatial imaging position in the inversion effectively increases the number of equa-

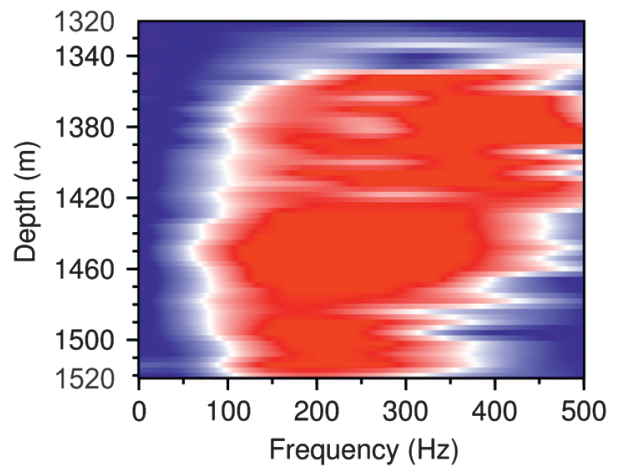

Figure 7. The amplitude spectrum of a shot gather at the depth of $1290 \mathrm{~m}$ in well A and receivers in well B. A starting frequency of $100 \mathrm{~Hz}$ is chosen for the inversion. tions without changing the number of unknown model parameters;

Because of the poor signal-to-noise ratio of the field data sets, the anisotropy parameter is assumed to be a constant, along with a spatially variable $2 \mathrm{D}$ horizontal velocity function. Figure 8 displays the velocity images reconstructed by waveform tomography at three different stages:

1) the velocity model after using the first group of frequency components $(100,102,104,106$, and $108 \mathrm{~Hz})$,

2) the velocity model after using six groups of frequency components $(100,102, \ldots, 158 \mathrm{~Hz})$, and

3 ) the final velocity model after using all 20 groups of frequency components $(100,102, \ldots, 298 \mathrm{~Hz})$.

Using multiple frequency bands during inversion does a lot more than suppress noise. It actually combines wavepaths with different this means the inverse problem being much better determined. 
model sensitivities in an inversion. Compared with traveltime tomography, waveform tomography generates a velocity model with a better character of layered structure, with continuity in the horizontal direction and high resolution in the vertical direction.

To confirm the results of waveform tomography for cases with such a poor ray coverage, we design a checkerboard resolution test. A checkerboard model (Figure 9a) is a superimposition of an alternating positive and negative anomaly pattern onto the final real data inversion model. The size of an anomaly pattern is $8 \mathrm{~m}$, which is smaller than the

a)

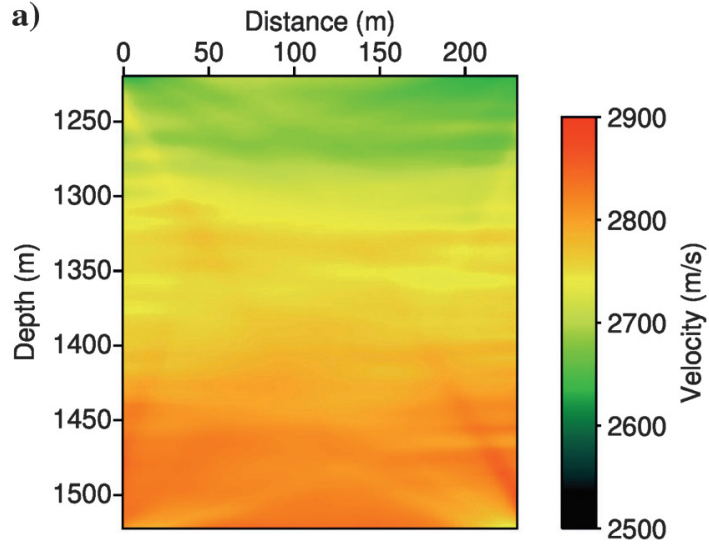

b)

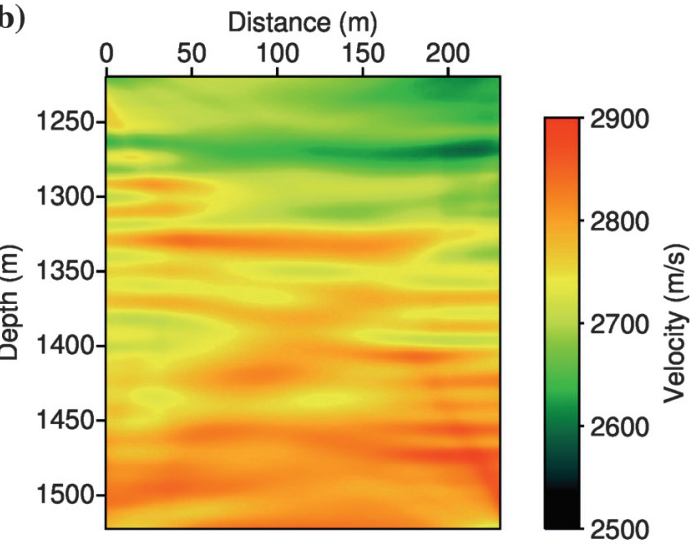

c)

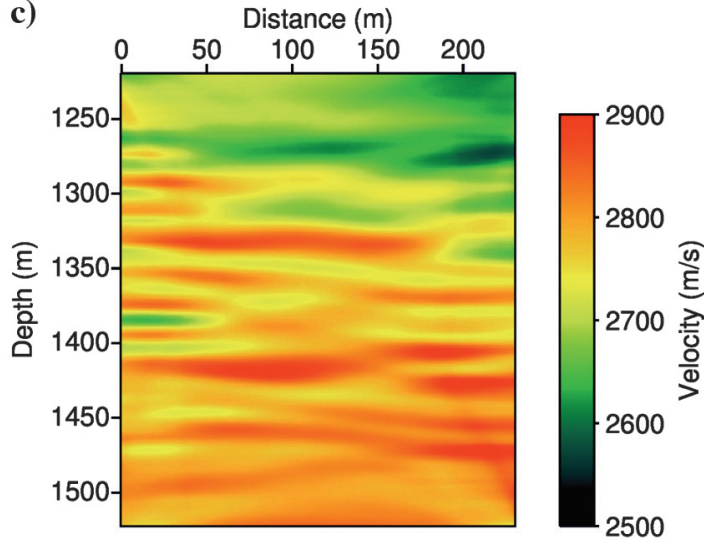

Figure 8. Velocity models reconstructed from waveform tomography. (a) Reconstructed velocity model after using the first group of frequency components $(100,102, \ldots, 108 \mathrm{~Hz})$. (b) Reconstructed velocity model after using six groups of frequency $(100,102, \ldots$, $158 \mathrm{~Hz}$ ). (c) Reconstructed velocity model after using all 20 groups of frequencies $(100,102, \ldots, 298 \mathrm{~Hz})$. size of most layered structures in the reconstructed velocity model (Figure $8 \mathrm{c}$ ). The anomaly pattern is $\pm 1 \%$ of the velocity model, which satisfies linear conditions in the iterative procedure of waveform inversion (Rao et al., 2006). We use this model to generate two synthetic data sets of crosshole seismic measurements, with exactly the same source and receiver geometry as that in the field.
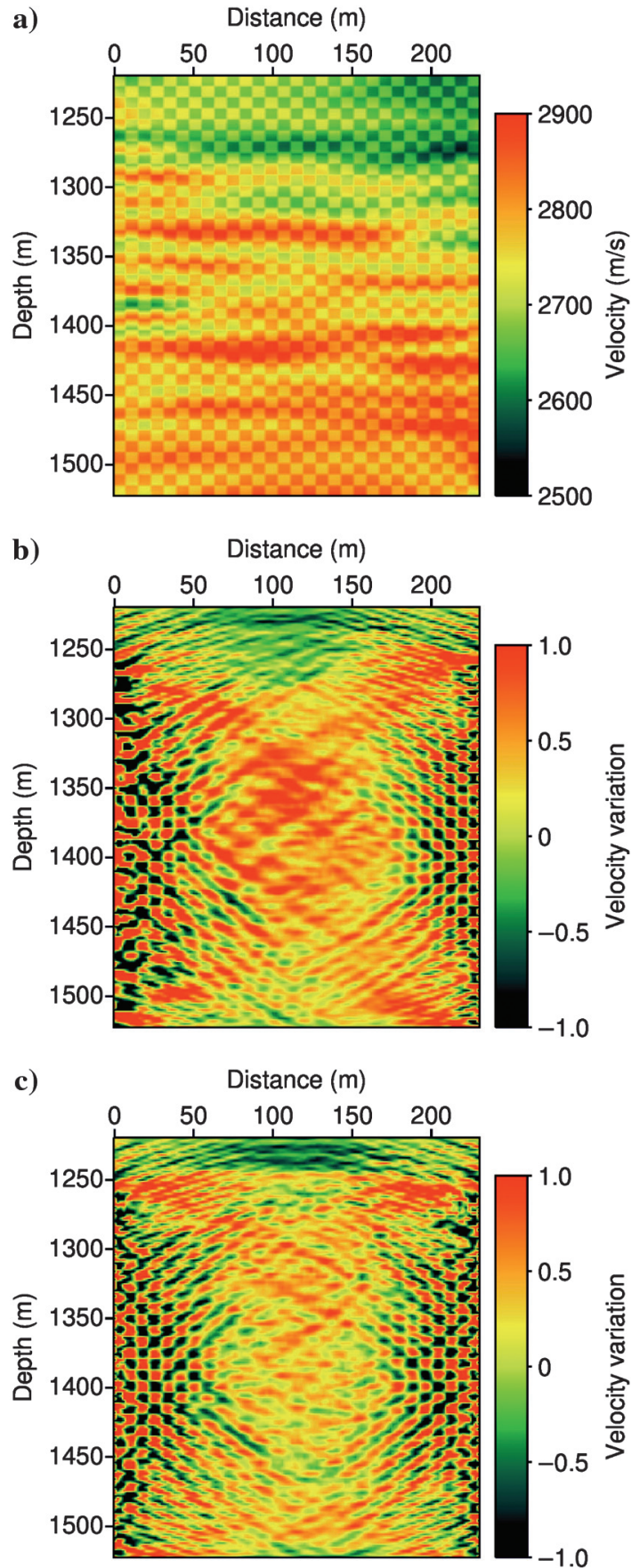

Figure 9. (a) A checkerboard velocity model used to generate two sets of synthetic seismic data, with the exact source-receiver configurations as the crosshole field measurement. (b) Normalized velocity variation in a reconstructed velocity model by waveform tomography using only one data set. (c) Normalized velocity variation in reconstructed velocity model by waveform tomography using both data sets simultaneously. 
When performing waveform tomography with synthetic data sets, we use exactly the same frequency components and the same inversion parameters as those used in the field data inversion example. We use the velocity model obtained from the field data inversion (Figure 8c) as the starting model.

We use either a single data set or two data sets together in the inversion tests. Figure $9 \mathrm{~b}$ and $9 \mathrm{c}$ shows the recovered velocity variation from the synthetic data inversion. To highlight the effectiveness, here we display velocity variations, rather than the velocities, in the two tomographic images. The variation pattern is the difference between the reconstructed velocity model and the start model, and it is normalized by the corresponding value in the start model.

When using a single data set in the inversion (Figure 9b), the left side region has been poorly recovered, compared with the right side region. This is because in the left side the raypaths are not evenly distributed (Figure 2a). When combining two measurements in the inversion (Figure 9c), because the rays are evenly distributed (Figure 2c), we can see that the inversion image has much better resolutions on the left and right sides, close to the boreholes.

\section{CONCLUSIONS}

To improve the ray coverage between two boreholes, the crosshole seismic acquisition has been designed in a cross-firing manner. Two crosshole seismic data sets, in the conventional sense, have been used jointly in traveltime and waveform tomography.

The sediment structure in the study area is featured with periodic thin layers. For this case, the velocity anisotropic effect should be considered in tomographic inversion. We have implemented a threestage tomographic inversion:

1) isotropic traveltime tomography to invert for the maximum horizontal velocity,

2) anisotropic traveltime tomography, inverting for the anisotropic parameter, normalized difference between the maximum horizontal velocity and the maximum vertical velocity, and

3) anisotropic waveform tomography with an anisotropic wave equation to refine the maximum horizontal velocity model.

In the implementation of an anisotropic wave equation, the anisotropic parameter, rather than a simple shrink factor, has been given explicitly in regular finite-differencing grids. Only when this VTI anisotropy is considered, could the first arrivals of the field observation be matched by synthetics in the waveform tomography. Resolution analysis has proven the effectiveness of the cross-firing geometry, whereas joint inversion of two crosshole data sets has resulted in a high-resolution image. Future work would involve reverse time migration of crosshole seismic data, using these inverted velocity and anisotropy models.

\section{ACKNOWLEDGMENTS}

The authors are grateful to the National Natural Science Foundation of China (grant no. 41474111) and the sponsors of the Centre for Reservoir Geophysics, Imperial College London, for supporting this research.

\section{REFERENCES}

Alkhalifah, T., 1998, Acoustic approximations for processing in transversely isotropic media: Geophysics, 63, 623-631, doi: 10.1190/1.1444361.
Backus, G. E., 1962, Long-wave elastic anisotropy produced by horizontal layering: Journal of Geophysical Research, 67, 4427-4440, doi: 10.1029/ JZ067i011p04427.

Berryman, J. G., 1989, Weighted least-squares criteria for seismic traveltime tomography: IEEE Transactions on Geoscience and Remote Sensing, 27, 302-309, doi: 10.1109/36.17671.

Cervený, V., 2001, Seismic ray theory: Cambridge University Press.

Chapman, C. H., and R. G. Pratt, 1992, Traveltime tomography in anisotropy media - I: Theory: Geophysical Journal International, 109, 1-19, doi: 10.1111/j.1365-246X.1992.tb00075.x.

Fomel, S., 2004, On anelliptic approximations for qP velocities in VTI media: Geophysical Prospecting, 52, 247-259, doi: 10.1111/j.1365-2478 .2004.00413.x.

Gassmann, F., 1964, Introduction to seismic traveltime methods in anisotropic media: Pure and Applied Geophysics, 58, 63-112, doi: 10.1007/ BF00879140.

Pratt, R. G., 1990, Inverse theory applied to multi-source cross-hole tomography, part II: Elastic wave-equation method: Geophysical Prospecting, 38, 311-329, doi: 10.1111/j.1365-2478.1990.tb01847.x.

Pratt, R. G., and C. H. Chapman, 1992, Traveltime tomography in anisotropy media - II: Application: Geophysical Journal International, 109, 20-37, doi: 10.1111/j.1365-246X.1992.tb00076.x.

Pratt, R. G., W. J. McGaughey, and C. H. Chapman, 1993, Anisotropy velocity tomography - A case study in a near-surface rock mass: Geophysics, 58, 1748-1763, doi: 10.1190/1.1443389.

Pratt, R. G., and M. S. Sams, 1996, Reconciliation of crosshole seismic velocities with well information in a layered sedimentary environment: Geophysics, 61, 549-560, doi: 10.1190/1.1443981.

Pratt, R. G., C. Shin, and G. J. Hicks, 1998, Gauss-Newton and full Newton methods in frequency-space seismic waveform inversion: Geophysical Journal International, 133, 341-362, doi: 10.1046/j.1365-246X.1998.00498.x.

Pratt, R. G., and R. M. Shipp, 1999, Seismic waveform inversion in the frequency domain, Part 2: Fault delineation in sediments using crosshole data: Geophysics, 64, 902-914, doi: 10.1190/1.1444598.

Pratt, R. G., and M. H. Worthington, 1990, Inverse theory applied to multisource cross-hole tomography, Part I: Acoustic wave-equation method: Geophysical Prospecting, 38, 287-310, doi: 10.1111/j.1365-2478.1990. tb01846.x.

Rao, Y., and Y. Wang, 2005, Crosshole seismic tomography: working solutions to issues in real data traveltime inversion: Journal of Geophysics and Engineering, 2, 139-146, doi: 10.1088/1742-2132/2/2/008.

Rao, Y., and Y. Wang, 2011, Crosshole seismic tomography including the anisotropy effect: Journal of Geophysics and Engineering, 8, 316-321, doi: 10.1088/1742-2132/8/2/016.

Rao, Y., Y. Wang, and J. Morgan, 2006, Crosshole seismic waveform tomography - II. Resolution analysis: Geophysical Journal International, 166, 1237-1248, doi: 10.1111/j.1365-246X.2006.03031.x.

Ravaut, C., S. Operto, L. Improta, J. Virieux, A. Herrero, and P. Dell'Aversana, 2004, Multiscale imaging of complex structures from multifold wide-aperture seismic data by frequency-domain full-waveform tomography - Application to a thrust belt: Geophysical Journal International, 159, 1032-1056, doi: 10.1111/j.1365-246X.2004.02442.x.

Sirgue, L., and R. G. Pratt, 2004, Efficient waveform inversion and imaging - A strategy for selecting temporal frequencies and waveform inversion: Geophysics, 69, 231-248, doi: 10.1111/j.1365-246X.2006.03030.x.

Thomsen, L., 1986, Weak elastic anisotropy: Geophysics, 51, 1954-1966, doi: 10.1190/1.1442051.

Wang, Y., 2011, Seismic waveform modeling and tomography, in H. K. Gupta, ed., Encyclopedia of solid earth geophysics: Springer Verlag, $1290-1301$.

Wang, Y., 2013, Simultaneous computation of seismic slowness paths and the traveltime field in anisotropic media: Geophysical Journal International, 195, 1141-1148, doi: 10.1093/gii/ggt278.

Wang, Y., 2014, Seismic ray tracing in anisotropic media: A modified Newton algorithm for solving highly nonlinear systems: Geophysics, 79, no. 1 , T1-T7, doi: 10.1190/geo2013-0110.1.

Wang, Y., and Y. Rao, 2006, Crosshole seismic waveform tomography - I. Strategy for real data application: Geophysical Journal International, 166, 1224-1236, doi: 10.1111/j.1365-246X.2006.03030.x.

Wang, Y., and Y. Rao, 2009, Reflection seismic waveform tomography: Journal of Geophysical Research, 114, B03304, doi: 10.1029/2008JB005916.

Warner, M., A. Ratcliffe, T. Nangoo, J. Morgan, A. Umpleby, N. Shah, V. Vinje, I. Stekl, L. Guasch, C. Win, G. Conroy, and A. Bertrand, 2013 , Anisotropic 3D full-waveform inversion: Geophysics, 78, no. 2, R59R80, doi: 10.1190/geo2012-0338.1.

Zhou, B., and S. A. Greenhalgh, 2003, Crosshole seismic inversion with normalized full-waveform amplitude data: Geophysics, 68, 1320-1330, doi: $10.1190 / 1.1598125$.

Zhou, B., S. Greenhalgh, and A. Green, 2008, Nonlinear traveltime inversion scheme for crosshole seismic tomography in tilted transversely isotropic media: Geophysics, 73, no. 4, D17-D33, doi: 10.1190/1.2910827. 